\title{
OPEN Blue to yellow emission from (Ga, In)/GaN quantum wells grown on pixelated silicon substrate
}

\author{
Benjamin Damilano ${ }^{1 凶}$, Marc Portail ${ }^{1}$, Eric Frayssinet ${ }^{1}$, Virginie Brändli ${ }^{1}$, Florian Faure ${ }^{2}$, \\ Christophe Largeron ${ }^{2}$, David Cooper ${ }^{2}$, Guy Feuillet ${ }^{2}$ \& Daniel Turover ${ }^{3}$
}

It is shown that substrate pixelisation before epitaxial growth can significantly impact the emission color of semiconductor heterostructures. The wavelength emission from $\ln _{x} G_{1-x} N / G a N$ quantum wells can be shifted from blue to yellow simply by reducing the mesa size from $90 \times 90 \mu^{2}$ to $10 \times 10$ $\mu \mathrm{m}^{2}$ of the patterned silicon used as the substrate. This color shift is mainly attributed to an increase of the quantum well thickness when the mesa size decreases. The color is also affected, in a lesser extent, by the trench width between the mesas. Cathodoluminescence hyperspectral imaging is used to map the wavelength emission of the $\ln _{x} \mathrm{Ga}_{1-x} \mathrm{~N} / \mathrm{GaN}$ quantum wells. Whatever the mesa size is, the wavelength emission is red-shifted at the mesa edges due to a larger quantum well thickness and In composition.

Group-III nitride semiconductors are the materials of choice for the fabrication of blue and white light emitting diodes ${ }^{1}$. The active region is made of blue light emitting $(\mathrm{Ga}, \mathrm{In}) \mathrm{N}$ quantum wells $(\mathrm{QWs})$ heterostructures encapsulated in a yellow/red emitting luminophore which converts part of these blue photons to generate white light. These devices are now the most efficient white light sources for general lighting ${ }^{2}$. However, the color of these lamps can only be weakly tuned. Fine control of the light emission over the visible range is only possible if colors can be controlled separately. Ideally, an independent control of blue, green and red colors opens up a wide color range. In principle, this can be done easily by mixing three different LEDs: for example (Ga,In) $\mathrm{N}$-based for blue and green, and (Al,Ga,In)P for red. However, with the aim of developing high resolution and high luminance micro-displays ${ }^{3,4}$, this approach is no longer possible. Mixing the three basic blue/green/red colors directly on the same wafer is therefore highly desirable and constitutes an important challenge. Efforts towards this objective have been reported in the literature using for example nanowires grown by molecular beam epitaxy ${ }^{5,6}$, light conversion by $(\mathrm{Ga}, \mathrm{In}) \mathrm{N}$ multiple quantum wells ${ }^{7}$, facets with different orientations ${ }^{8}$, and local etching of red-emitting LED structures ${ }^{9}$.

Here, we propose the use of mesa-patterned silicon substrates to tune the light emission of (Ga,In)N quantum wells. Mesa-patterned silicon substrates are known to be an efficient way to avoid crack formation in thick $\mathrm{GaN}$ structures ${ }^{10-13}$. This is achieved by stress relaxation at the mesa edges that compensate the tensile strain in the GaN layers grown on silicon arising from the thermal expansion coefficient mismatch between $\mathrm{GaN}$ and silicon ${ }^{10-13}$. Mesa-patterned silicon substrates have been successfully exploited for the fabrication of devices such as efficient visible LEDs on silicon substrate ${ }^{14}$, laser diodes ${ }^{15}$, exciton-polariton laser ${ }^{16}$, LEDs incorporating a high reflectivity distributed light reflector ${ }^{17}$, high electron mobility transistors ${ }^{18}$. Xu et al. reported an enhanced indium incorporation in $(\mathrm{Ga}, \mathrm{In}) \mathrm{N}$ QWs at the corner of $340 \times 340 \mu \mathrm{m}^{2}$ mesas grown on patterned silicon substrate ${ }^{19}$ but $^{2}$ mesa-patterned silicon substrates have never been studied with the objective of modifying the emission color as a function of the mesa size.

\section{Results}

In order to determine the impact of substrate patterning, the micro-photoluminescence was measured at room temperature at the center of 24 different mesas. The sample is divided into different regions with a surface area of $4 \times 6 \mathrm{~mm}^{2}$. Each region contains a square array of square mesas, each with a side length $\mathrm{L}$ and a trench width W. There are 6 different mesas sizes: $\mathrm{L}=10,20,30,40,60,90 \mu \mathrm{m}$ and 4 different trench widths $\mathrm{W}=10,13,30$,

${ }^{1}$ Université Côte D'Azur, CNRS, CRHEA, Rue B. Gregory, Valbonne, France. ${ }^{2}$ Univ. Grenoble Alpes, CEA, LETI, 38000 Grenoble, France. ${ }^{3}$ SILSEF, 382 Rue Louis Roustin, 74160 Archamps, France. ${ }^{\varpi}$ email: bd@crhea.cnrs.fr 

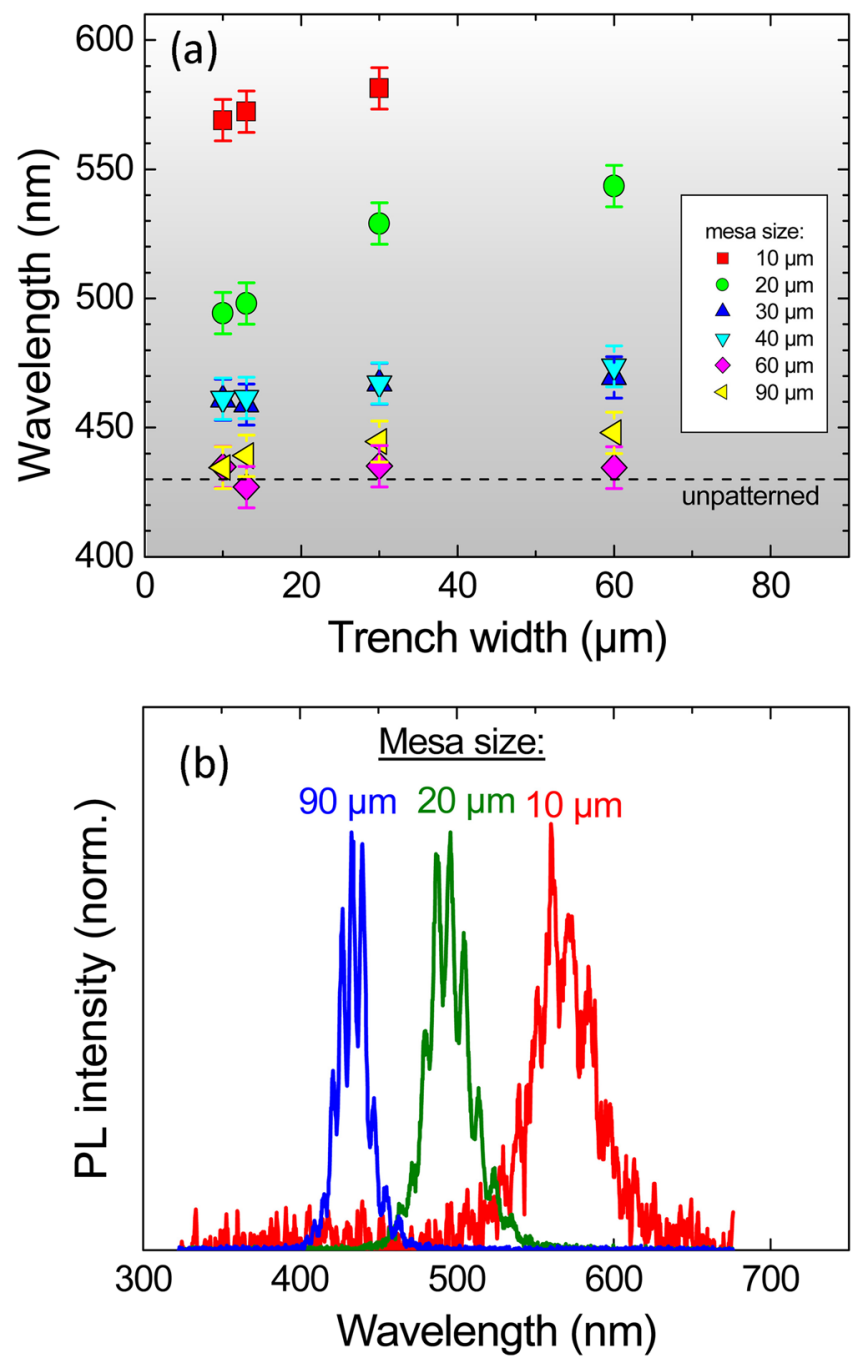

Figure 1. (a) Room temperature micro-photoluminescence peak wavelength from the InGaN/GaN multiple quantum well at the center of the mesas with variable sizes and trench widths. The dashed line corresponds to the emission at the center of the reference sample without patterns. (b) Examples of spectra with normalized intensities obtained for variable mesa sizes $\mathrm{L}=10,20$, and $90 \mu \mathrm{m}$ with a constant trench width of $10 \mu \mathrm{m}$ (mesas L10W10, L20W10, and L90W10).

$60 \mu \mathrm{m}$, totalizing 24 different regions. In the following the mesas are identified by LxWy, where $\mathrm{x}$ is the side length and $y$ the trench width in $\mu \mathrm{ms}$. The peak wavelength of the (Ga,In)N/GaN MQW extracted from a Gaussian fit of the PL spectra is shown in Fig. 1a. Some of the spectra are given as an example in Fig. 1b for different mesa sizes and a trench width of $10 \mu \mathrm{m}$. Note that the PL spectra are structured by oscillations coming from the interference of light in the cavity formed by the nitride layers sandwiched by the air and the silicon substrate. The main result shown by the Fig. 1a is that the PL wavelength increases from 430 to $440 \mathrm{~nm}$ for the largest mesa sizes of $60-90 \mu \mathrm{m}$ to $\sim 570-580 \mathrm{~nm}$ for the smallest mesa size of $10 \mu \mathrm{m}$. This shows that simply by structuring the substrate, different color emissions (at least from blue to green and yellow) can be monolithically integrated on the same sample in one single growth run. The wavelength emission also increases when the trench width increases however to a lesser extent than for the dependence observed as a function of the mesa size. This variation is the largest for the mesas with side length $\mathrm{L}=20 \mu \mathrm{m}$ (Fig. 1a): the peak PL wavelength shifts from $494 \mathrm{~nm}$ (blue-green) to $544 \mathrm{~nm}$ (green) when varying the trench width from 10 to $60 \mu \mathrm{m}$. Some variation is found in the data, for example the QW emission of L60W60 is at slightly shorter wavelength than the one of L90W60. This is due to the non-perfect homogeneity of the QW on the 2-inch wafer during the growth (see Supplementary Information, Figure S1).

The integrated photoluminescence intensity from the center of the mesas as a function of the peak wavelength emission follows an asymmetric bell shape curve. It is maximum in the $470-500 \mathrm{~nm}$ wavelength range and decreases by a factor 2.5 at $430 \mathrm{~nm}$ and by a factor 100 at $580 \mathrm{~nm}$. The PL signal of the mesa L10W60 is too weak for a reliable estimation of the peak wavelength emission.

Cathodoluminescence (CL) mapping of the mesas was used to obtain spatially resolved spectra from each mesa type, with a step size of $700 \mathrm{~nm}$. One example is shown in Fig. 2, where a mesa L40W30 is investigated 


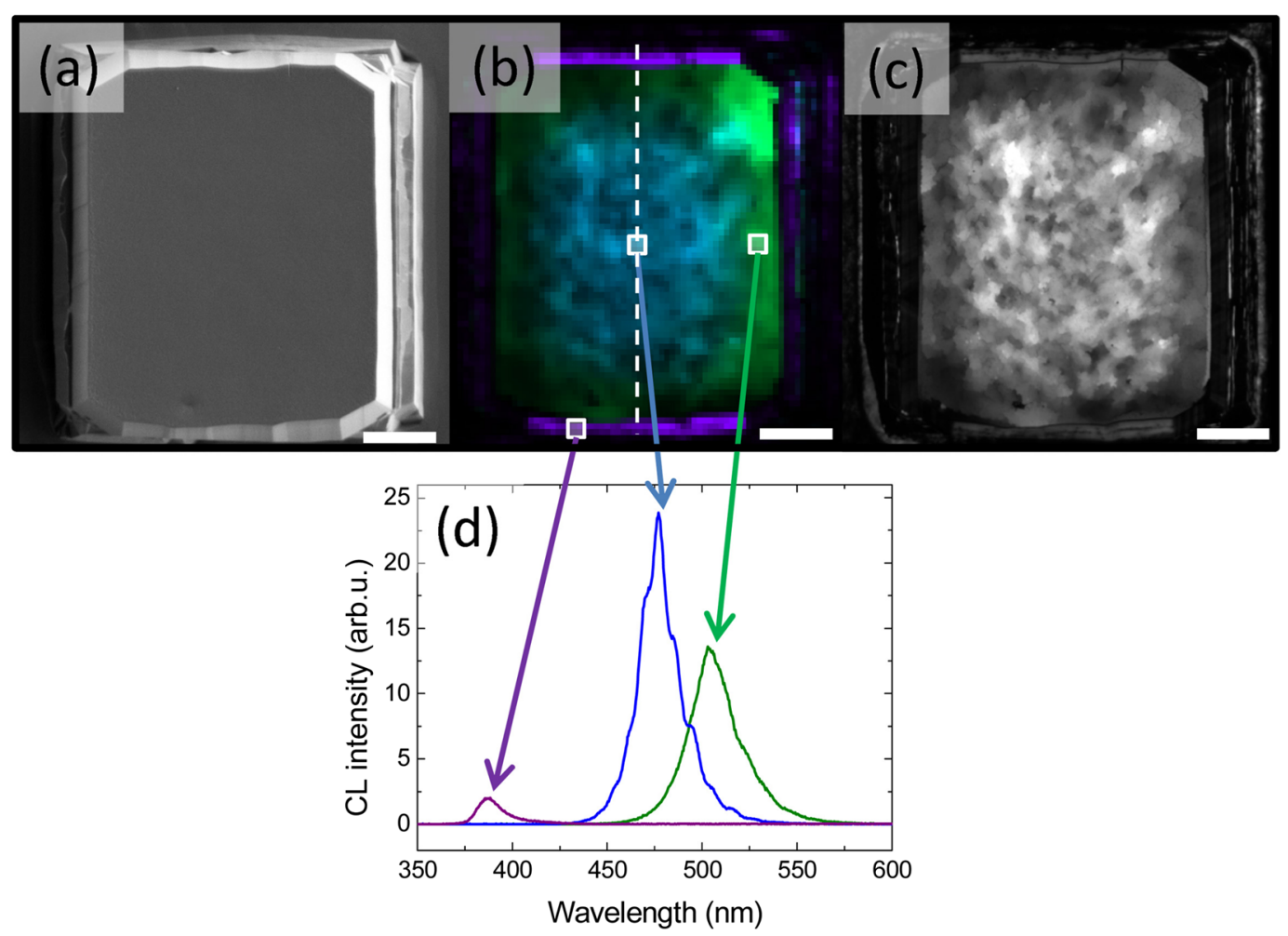

Figure 2. (a) Scanning electron microscopy image of a mesa with a side length of $L=40 \mu \mathrm{m}$ and a trench width $\mathrm{W}=30 \mu \mathrm{m}$. (b) Cathodoluminescence map of the same mesa. The regions corresponding to a wavelength of 390, 475, and $510 \mathrm{~nm}$ (spectral width of $10 \mathrm{~nm}$ ) are highlighted in violet, blue, and green, respectively. (c) Corresponding cathodoluminescence panchromatic image. (d) Characteristic examples of the spectra obtained in three different regions of the mesa: center, edge, and side facet. The white scale bar in (a), (b) and (c) is $10 \mu \mathrm{m}$.

(scanning electron microcopy (SEM) image in Fig. 2a). Figure $2 b$ shows the CL intensity map for 3 different wavelengths: $390 \mathrm{~nm}, 475 \mathrm{~nm}$ and $520 \mathrm{~nm}$ (corresponding to the violet, blue and green colors). The spectra recorded at 3 different positions on the mesas are shown in Fig. 2d. Blue CL emission is observed at the center of the mesa while the color is shifting towards the green at the edge of the mesa. Violet emission is observed on the inclined facets located at the edges of the GaN mesa. The CL spectra extracted at the center of the mesa following the white dotted line in Fig. 2b are shown in Fig. 3g. This profile indicates that the PL emission is rather homogeneous at the center of the mesa and shifts to longer wavelength at $\sim 10 \mu \mathrm{m}$ from the mesa edge. The panchromatic image of Fig. $2 c$ indicates a slightly weaker intensity at the edges of the $\mathrm{GaN}$ mesa. This decrease in the internal quantum efficiency is the typical evolution expected from $\operatorname{In}_{\mathrm{x}} \mathrm{Ga}_{1-\mathrm{x}} \mathrm{N} / \mathrm{GaN}$ MQWs when the wavelength increases ${ }^{20,21}$.

The CL mapping experiments have been repeated on different mesas and in order to reduce the time required for these measurements, only line scan analysis at the center of the mesas were performed. The results are shown in Fig. 3a-h for mesas L20W10, L20W13, L20W30, L20W60, L40W10, L40W13, L40W30, and L40W60. As reported above for $\mu \mathrm{PL}$ experiments, the peak CL wavelength at the mesa center shifts towards longer wavelength when the mesa size decreases and the trench width between mesas increases. For all the mesas investigated, the peak CL wavelength at the mesa edges is red-shifted compared to the value at the center of the mesa.

\section{Discussion}

The origin of the large emission red-shift when the mesa size decreases and the trench width increases can be explained. Firstly, the fundamental energy of the $\mathrm{e}_{1}-\mathrm{hh}_{1}$ excitonic transition of the $\operatorname{In}_{\mathrm{x}} \mathrm{Ga}_{1-\mathrm{x}} \mathrm{N} / \mathrm{GaN}$ quantum well is given by the following expression ${ }^{22}$ :

$$
E_{\mathrm{QW}}(x, h)=E_{g}(x)+e_{1}(x, h)+h h_{1}(x, h)-R y(x, h)-e F(x) h,
$$

where $E_{g}$ is the bandgap energy of $\operatorname{In}_{x} G_{1-x} N, e_{1}$ and $h h_{1}$ are the confinement energies of the electron and hole, Ry is the exciton binding energy, $\mathrm{F}$ is the internal electric field, $\mathrm{e}$ is the electron charge, and $\mathrm{h}$ is the quantum well thickness.

A red shift of the emission could be due to an increased Indium composition. Indeed, as the In composition $x$ increases the emission energy of the QW decreases because the bandgap of $\operatorname{In}_{\mathrm{x}} \mathrm{Ga}_{1-\mathrm{x}} \mathrm{N}$ narrows and in addition the internal electric field increases. Similarly, an increase of the QW thickness can cause a decrease of $\mathrm{E}_{\mathrm{QW}}$, mainly due to the term -eFh in (1). The strain state of the $\operatorname{In}_{\mathrm{x}} \mathrm{Ga}_{1-\mathrm{x}} \mathrm{N}$ QWs can cause a change in the bandgap energy $\mathrm{E}_{\mathrm{g}}$ and also of the internal piezoelectric field $\mathrm{F}$. Indeed, strain relaxation at the mesa edges can cause a PL 


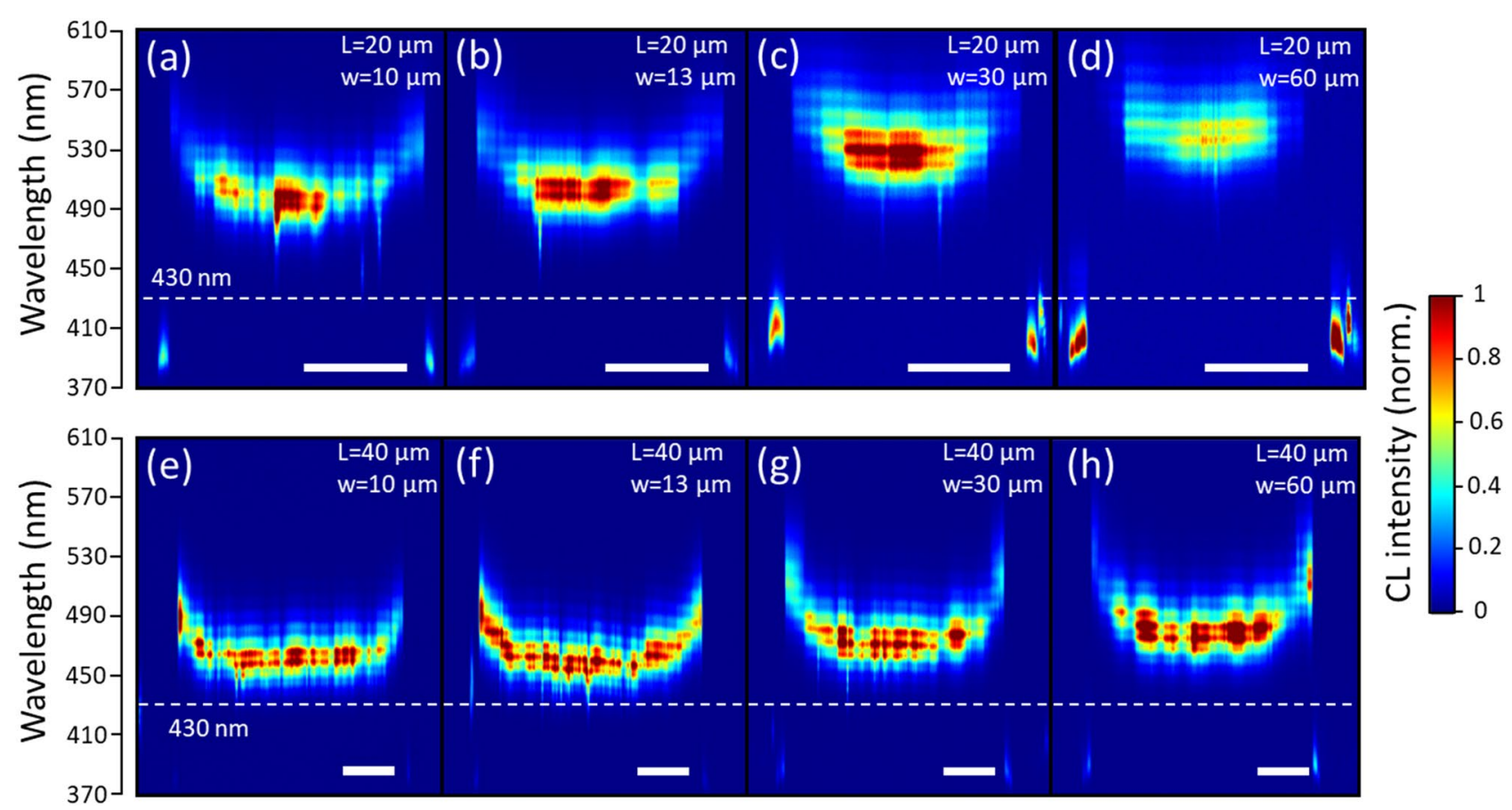

Figure 3. Room temperature cathodoluminescence spectra along a line in the middle of the mesas with a side length $\mathrm{L}=20 \mu \mathrm{m}(\mathbf{a}-\mathbf{d})$ or $\mathrm{L}=40 \mu \mathrm{m}(\mathbf{e}-\mathbf{h})$. The width in between mesas is $\mathrm{W}=10 \mu \mathrm{m}(\mathbf{a}, \mathbf{e}), 13 \mu \mathrm{m}(\mathbf{b}, \mathbf{f}), 30 \mu \mathrm{m}$ $(\mathbf{c}, \mathbf{g}), 60 \mu \mathrm{m}(\mathbf{d}, \mathbf{h})$. The intensity (normalized) of the spectra is color coded from blue (0) to red (1). The doted white line corresponds to a wavelength of $430 \mathrm{~nm}$,as obtained for the unpatterned substrate. The white scale bar is $10 \mu \mathrm{m}$ in all figures.

blue-shift of the $\operatorname{In}_{x} \mathrm{Ga}_{1-x} \mathrm{~N}$ QWs due to a reduction of the internal piezoelectric field. This last effect has been shown to have a significant impact for very small nanostructures: the PL emission of red-emitting $\operatorname{In}_{\mathrm{x}} \mathrm{Ga}_{1-\mathrm{x}} \mathrm{N}$ QWs is shifted to green and blue when they are selectively etched to form nanowires with diameters of 150 and $50 \mathrm{~nm}$, respectively ${ }^{9}$. In our work, the minimum mesa size is $10 \mu \mathrm{m}$ and therefore a strong impact of strain variation on the $\operatorname{In}_{\mathrm{x}} \mathrm{Ga}_{1-\mathrm{x}} \mathrm{N}$ bandgap and the piezoelectric field at the center of the mesas is not expected.

As described above and shown in Fig. 1b, the PL spectra are structured by interference fringes. The spacing of these fringes is characteristic of the total nitride layer thickness grown on the silicon substrate ${ }^{23}$. At a wavelength around $460 \mathrm{~nm}$, at the mesa center, this spacing is estimated to be $8.3 \mathrm{~nm}$ and $6.3 \mathrm{~nm}$ for the mesas L90W10 and L20W10, respectively. This clearly shows that the total thickness of the nitride layers is larger for L20W10 compared to L90W10.

In order to obtain direct and more quantitative data, TEM experiments were conducted to measure the $\mathrm{In}_{\mathrm{x}} \mathrm{Ga}_{1-\mathrm{x}} \mathrm{N}$ QW thicknesses. Figure 4a shows cross section HAADF STEM images at the top of a mesa with a side length of $20 \mu \mathrm{m}$ and a trench width of $60 \mu \mathrm{m}$ (L20W60) showing the $\operatorname{In}_{\mathrm{x}} \mathrm{Ga}_{1-\mathrm{x}} \mathrm{N}$ quantum wells at different positions. Figure $4 \mathrm{~b}$ shows a higher magnification HAADF STEM image at the mesa edge and Fig. $4 \mathrm{c}$ shows a corresponding EDX map. Figure 4d shows a HAADF STEM image acquired $2 \mu \mathrm{m}$ away from the mesa edge and Fig. 4e the corresponding EDX map. These experiments were repeated at other positions of the mesa L20W60 and also for mesa L20W10. The QW thicknesses were extracted from all these images and are shown in Fig. $4 \mathrm{f}$ as a function of the distance from the mesa edge. $\operatorname{The} \operatorname{In}_{\mathrm{x}} \mathrm{Ga}_{1-\mathrm{x}} \mathrm{N}$ QW thickness tends to a constant at the center of the mesa of about $2.9 \mathrm{~nm}$ and $4.5 \mathrm{~nm}$, for L20W10 and L20W60, respectively. Other thickness measurements were performed by TEM on a mesa L10W10. The $\operatorname{In}_{x} \mathrm{Ga}_{1-\mathrm{x}} \mathrm{N}$ QW thickness at the center of this mesa is $5 \mathrm{~nm}$ (Fig. S2 of Supplementary Information). These values are significantly larger than the nominal $\operatorname{In}_{\mathrm{x}} \mathrm{Ga}_{1-\mathrm{x}} \mathrm{N}$ QW thickness of $2 \mathrm{~nm}$ (on a planar substrate). Calculations of the $\mathrm{e}_{1}-\mathrm{hh}_{1}$ fundamental transition of the $\operatorname{In}_{\mathrm{x}} \mathrm{Ga}_{1-\mathrm{x}} \mathrm{N} /$ GaN quantum well were performed to determine whether the thickness variation can account for the wavelength shift at the center of the mesas. This calculation was performed using the nominal indium content of 0.13 and the other parameters (bandgap, piezoelectric constants,...) were taken from Ref. ${ }^{24}$ and summarized in Table S1 of Supplementary Information. The calculated QW emission wavelengths are 430, 460, 525, and $551 \mathrm{~nm}$ for QW thicknesses of 2, 2.9, 4.5, and $5 \mathrm{~nm}$, respectively. Therefore, this QW thickness increase can account for a large part the observed red-shift of the QW emission energy at the mesa center when the mesa size decreases.

EDX measurements were performed at the edge of mesas L20W10 and L10W10 and give an average In composition of $13.8 \pm 2.0 \%$ and $18.6 \pm 2.0 \%$, respectively. This increase of the In composition can also explain the redshift of the photoluminescence emission of the mesas when their size decreases. The exact quantification of these EDX measurements in such small QW structures is complicated. However, all the observations were made using identical experimental conditions and quantification, such as the relative values can be compared. The difference in In concentration is also confirmed by the increase in intensity measured by HAADF in the InGaN wells. 

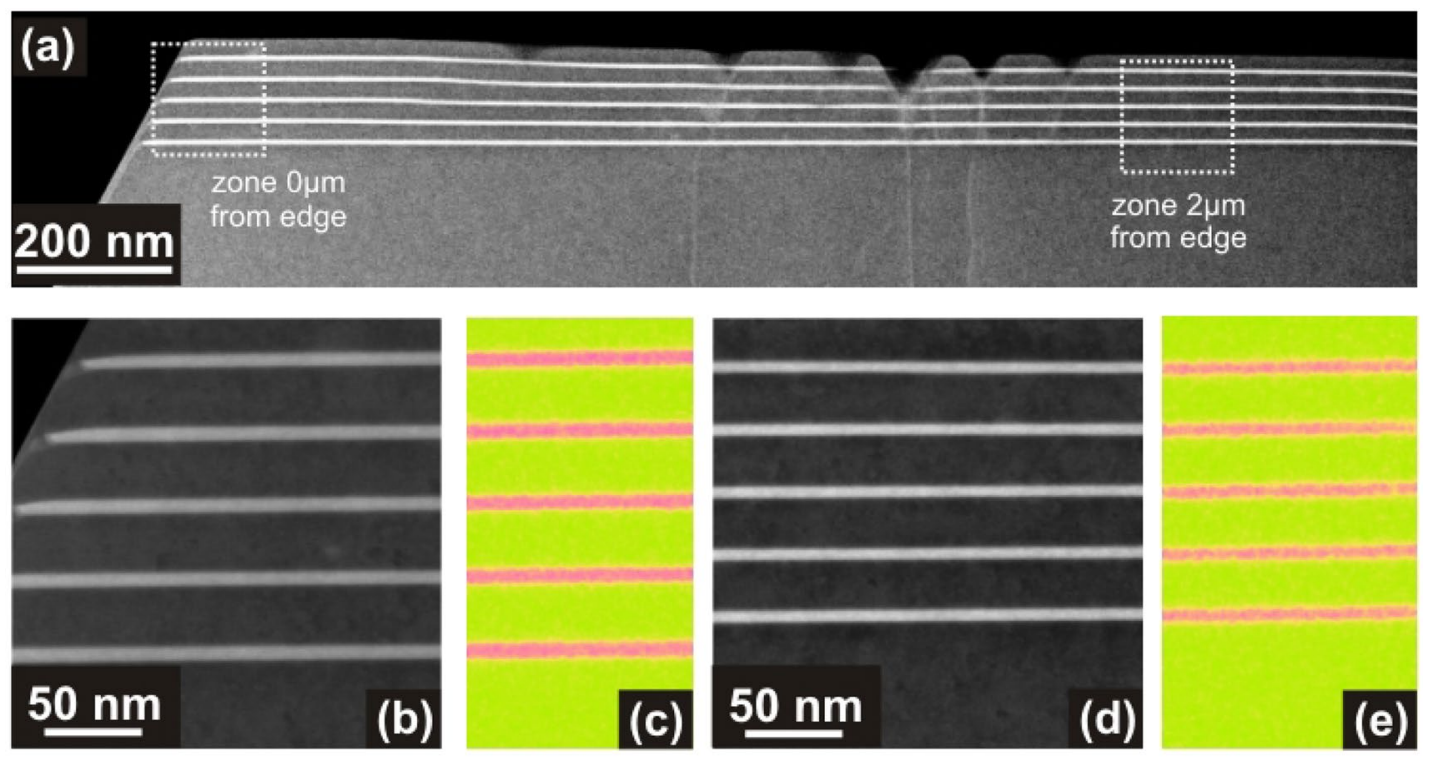

(f)
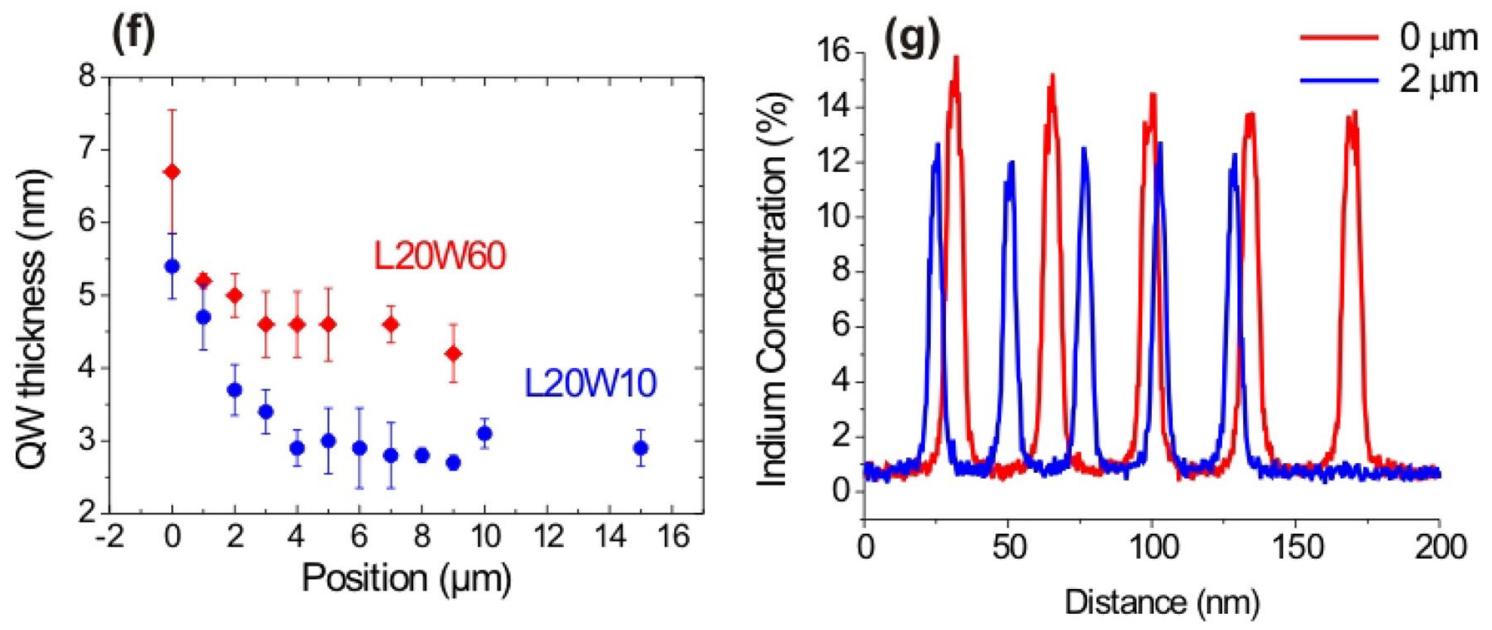

Figure 4. (a) HAADF STEM cross section images taken in the top area of a mesa L20W60 (side length of $20 \mu \mathrm{m}$ and trench width of $60 \mu \mathrm{m}$ ). (b) shows a higher magnification STEM image at the mesa edge and (c) an EDX map showing the InGaN layers. (d) A higher magnification STEM image acquired $2 \mu \mathrm{m}$ away from the mesa edge and (e) an EDX map showing the InGaN layers. (f) The InGaN quantum well thicknesses extracted from the transmission electron microscopy images as a function of the position ( 0 corresponds to the mesa edge) for mesa with a side length of $20 \mu \mathrm{m}$ and a trench width of $60 \mu \mathrm{m}$ (in red) or $10 \mu \mathrm{m}$ (in blue). (g) Quantitative profiles of the Indium content extracted from the EDX maps both at the edge and $2 \mu \mathrm{m}$ away from the edge for the mesa L20W60.

The HAADF STEM images indicate a strong increase of the QW thicknesses above a few micrometers from the mesa edges. This is again in good agreement with the wavelength redshifts observed close to the mesa edges for all the CL spectra shown in Fig. 3. More specifically for the mesas L20W10 and L20W60, the CL peak wavelength of the $\mathrm{In}_{\mathrm{x}} \mathrm{Ga}_{1-\mathrm{x}} \mathrm{N} / \mathrm{GaN}$ MQW also starts to shift at a few micrometers from the mesa edge as shown in Fig. 3a,d. To evaluate the variation of the indium composition of the $\operatorname{In}_{\mathrm{x}} \mathrm{Ga}_{1-\mathrm{x}} \mathrm{N}$ QWs the EDX measurements that were performed on the mesa L20W60 are shown in Fig. 4g. The quantitative In composition measurements show an average value of $13.8 \pm 2.0 \%$ at the edge and $11.6 \pm 2.0 \%$ at a position of $2 \mu \mathrm{m}$ from the edge. Indeed, this composition increase also contributes to the wavelength emission red-shift of the $\operatorname{In}_{\mathrm{x}} \mathrm{Ga}_{1-\mathrm{x}} \mathrm{N} / \mathrm{GaN}$ MQW at the mesa edges.

The enhanced growth at the edges is similar to the observations made by Honda et al. ${ }^{12}$ and attributed to the mass transport of chemical species from the $\mathrm{SiO}_{2}$ mask to the mesa edges. Honda et al. observed a strong GaN thickness variation between the center and the edge of the mesas for a mesa spacing larger than $50 \mu \mathrm{m}$, while it decreases down to $10 \%$ when the spacing between mesas is $10 \mu \mathrm{m}$ and the mesa size $200 \mu \mathrm{m}$. However, in our case, growth occurs in the trenches between the mesas and the growth enhancement should be attributed to another effect such as a perturbation of the critical layer in the gas phase close to the mesa edges ${ }^{25}$. The slightly larger In incorporation at the mesa edges can be related to a stress relaxation effect by the free edges. Indeed, the $\mathrm{In}_{\mathrm{x}} \mathrm{Ga}_{1-\mathrm{x}} \mathrm{N}$ layer grown close to the mesa edge can be submitted to a lower compressive stress compared to 

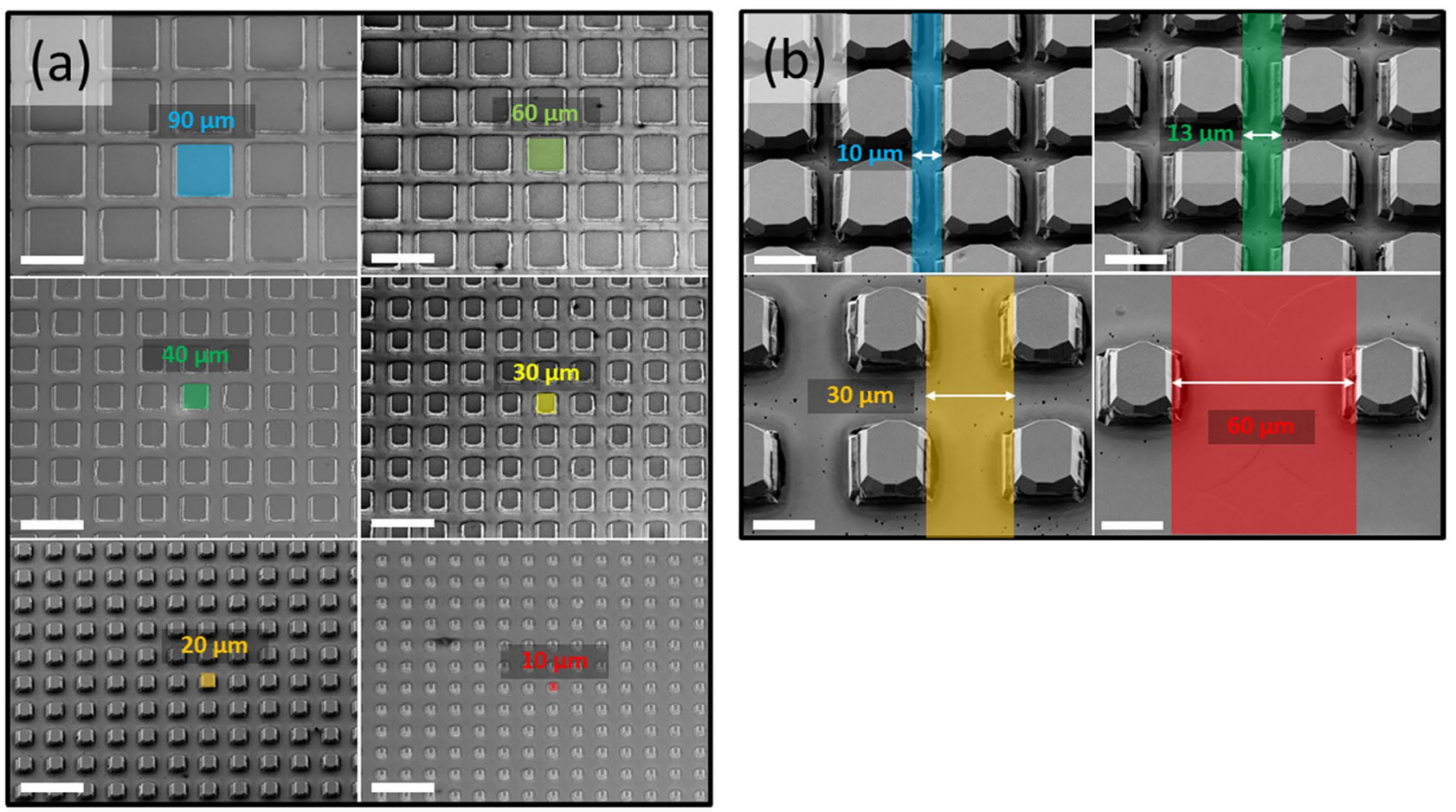

Figure 5. Scanning electron microscopy images at a tilted view of $30^{\circ}$ of mesas with variable sizes $L=10,2030$, 40 , and $60 \mu \mathrm{m}$ and a constant trench width $\mathrm{W}=30 \mu \mathrm{m}(\mathbf{a})$ and mesas with a constant size $\mathrm{L}=20 \mu \mathrm{m}$ and variable trench widths $\mathrm{W}=10,13,30$, and $60 \mu \mathrm{m}(\mathbf{b})$. The scale bar is respectively 100 and $20 \mu \mathrm{m}$ for (a) and (b).

the one at the center of the mesa and this reduction of stress can induce a larger In incorporation as shown for $\mathrm{In}_{\mathrm{x}} \mathrm{Ga}_{1-\mathrm{x}} \mathrm{N}$ quantum wells grown on relaxed ( $\left.\mathrm{Ga}, \mathrm{In}\right) \mathrm{N}$ layers ${ }^{26,27}$.

\section{Conclusion}

We have shown that the wavelength emission from an $\operatorname{In}_{x} \mathrm{Ga}_{1-\mathrm{x}} \mathrm{N} / \mathrm{GaN}$ MQW grown on a micro-pixelated $\mathrm{Si}$ substrate can be tuned from blue $(430 \mathrm{~nm})$ to yellow $(580 \mathrm{~nm})$ depending on the pixel size and the trench width between the pixels. Decreasing the pixel size and increasing the trench width leads to a longer wavelength emission for mesas with a side length smaller than $40 \mu \mathrm{m}$. This effect is mainly due to an increase of the QW thickness as the mesa side length decreases. The emission is relatively homogeneous at the center of the mesa but a red shift is observed near the mesa edges, again due to an increase of the QW thickness. The efficiency of the yellowemitting mesas is much smaller than for blue and green. Further optimization is required to get blue, green and red colors on the same wafer. The mesa sizes, pitches have to be finely tuned as a function of QW thickness and indium composition. This approach should then become promising for the fabrication of monolithic full-color micro-display emitters.

\section{Methods}

Patterned silicon substrate fabrication. The 2-inch $\mathrm{Si}(111)$ substrates were covered with a hard mask made of a tri-layer $\mathrm{SiO}_{2}-\mathrm{Al}-\mathrm{SiO}_{2}$ and subsequently patterned by direct laser photolithography using an Heidelberg MGP 1 machine. The Si mesas were etched using fluorine (SF6) based inductively coupled plasma (ICP) to provide high aspect ratio silicon etching.

The sample is divided into different regions with a surface area of $4 \times 6 \mathrm{~mm}^{2}$. Each region contains a square array of square mesas, each with a side length $\mathrm{L}$ and a trench width $\mathrm{W}$. There are 6 different mesas sizes: $\mathrm{L}=10$, 20, 30, 40, 60, $90 \mu \mathrm{m}$ and 4 different trench widths $\mathrm{W}=10,13,30,60 \mu \mathrm{m}$, totalizing 24 different regions. The trench depth in the silicon substrate is $10 \mu \mathrm{m}$. SEM images of some of the mesas obtained after growth are shown in Fig. 5.

Epitaxial growth. The samples were grown on the patterned $\mathrm{Si}(111)$ substrates as described above using low-pressure metal organic vapor phase epitaxy (MOVPE) in a commercial close coupled $7 \times 2$-in. showerhead reactor. The growth rates of the different layers are monitored in situ by a laser reflectivity set-up. Trimethylaluminium, trimethylgallium (for GaN growth), triethylgallium (for InGaN growth) and trimethylindium are used as group-III precursors while ammonia is used as group-V precursor. The structure of the samples comprises (starting from the substrate) a $220-\mathrm{nm}$ thick AlN buffer layer, a $2 \mu \mathrm{m}$-thick GaN layer and a 5 periods $\mathrm{In}_{0.13} \mathrm{Ga}_{0.87} \mathrm{~N}(2 \mathrm{~nm}) / \mathrm{GaN}(12 \mathrm{~nm})$ multiple quantum well (MQW). More details about the nucleation procedure on the silicon substrate can be found in Ref. ${ }^{28}$. The first stage of the GaN layer is grown in a 3-dimensional growth mode to reduce the threading dislocation density. On a separate sample grown without the MQW on 
patterned silicon, a threading dislocation density of $1-2 \times 10^{9} \mathrm{~cm}^{-2}$ was found emerging at the surface as measured by atomic force microscopy. The room temperature photoluminescence of such QW samples on un-patterned silicon (this sample was grown in the same growth run than the sample on patterned silicon substrate) is centred at $430 \mathrm{~nm}$.

Characterization. The surface of the samples was imaged by scanning electron microscopy. The microphotoluminescence $(\mu \mathrm{PL})$ was measured at room temperature using the $244 \mathrm{~nm}$ line of a frequency doubled Argon laser with a power density of $10 \mathrm{~W} / \mathrm{cm}^{2}$. The laser spot diameter is $\sim 2 \mu \mathrm{m}$. Cathodoluminescence (CL) was also performed at room temperature both in panchromatic and spatially and spectrally resolved modes.

High resolution scanning transmission electron microscopy (STEM) was performed using a probe corrected FEI Themis operated at $200 \mathrm{kV}$. Thin specimens were prepared using focused ion beam (FIB) milling in a FEI Strata 400 dual beam operated at $16 \mathrm{kV}$ to reduce specimen damage. The specimens were then cleaned using $2 \mathrm{kV}$ ions. On the different specimens, high-angle annular dark field (HAADF) STEM was performed on the same mesa structures looking down both the 0120 and 0110 zone axis. The indium content in the specimens was evaluated by comparing the relative intensity of the HAADF signal which is sensitive to the $\mathrm{Z}$ number in the specimens. In addition, energy dispersive X-ray spectroscopy (EDX) was performed in order to retrieve the relative indium concentrations. Quantification of the In concentration was performed using the Cliff-Lorimer method $^{29}$. Although it is difficult to quantify at these small dimensions using EDX, we assume that the relative concentrations in the same specimens that are measured using the same TEM settings, can be interpreted qualitatively.

Received: 5 May 2020; Accepted: 19 October 2020

Published online: 03 November 2020

\section{References}

1. Nakamura, S. Nobel Lecture: Background story of the invention of efficient blue InGaN light emitting diodes. Rev. Mod. Phys. 87, 1139-1151 (2015)

2. Narukawa, Y., Ichikawa, M., Sanga, D., Sano, M. \& Mukai, T. White light emitting diodes with super-high luminous efficacy. J. Phys. Appl. Phys. 43, 354002 (2010).

3. Jiang, H. X., Jin, S. X., Li, J., Shakya, J. \& Lin, J. Y. III-nitride blue microdisplays. Appl. Phys. Lett. 78, 1303 (2001).

4. Templier, F. GaN-based emissive microdisplays: a very promising technology for compact, ultra-high brightness display systems: GaN-based emissive microdisplays. J. Soc. Inf. Disp. 24, 669-675 (2016).

5. Kishino, K., Nagashima, K. \& Yamano, K. Monolithic integration of InGaN-based nanocolumn light-emitting diodes with different emission colors. Appl. Phys. Express 6, 012101 (2013).

6. Ra, Y.-H. et al. Full-color single nanowire pixels for projection displays. Nano Lett. 16, 4608-4615 (2016).

7. Damilano, B. et al. Metal organic vapor phase epitaxy of monolithic two-color light-emitting diodes using an InGaN-based light converter. Appl. Phys. Express 6, 092105 (2013).

8. Funato, M. et al. Experimental and theoretical considerations of polarization field direction in semipolar InGaN/GaN quantum wells. Appl. Phys. Express 3, 071001 (2010).

9. Chung, K., Sui, J., Demory, B. \& Ku, P.-C. Color mixing from monolithically integrated InGaN-based light-emitting diodes by local strain engineering. Appl. Phys. Lett. 111, 041101 (2017).

10. Zamir, S., Meyler, B. \& Salzman, J. Thermal microcrack distribution control in GaN layers on Si substrates by lateral confined epitaxy. Appl. Phys. Lett. 78, 288 (2001).

11. Dadgar, A. et al. Crack-Free InGaN/GaN Light Emitters on Si(111). Phys. Status Solidi A 188, 155-158 (2001).

12. Honda, Y., Kuroiwa, Y., Yamaguchi, M. \& Sawaki, N. Growth of GaN free from cracks on a (111)Si substrate by selective metalorganic vapor-phase epitaxy. Appl. Phys. Lett. 80, 222-224 (2002).

13. Hossain, T. et al. Stress distribution of $12 \mu \mathrm{m}$ thick crack free continuous GaN on patterned Si(110) substrate. Phys. Status Solidi C 10, 425-428 (2013).

14. Tao, X. et al. Performance enhancement of yellow InGaN-based multiple-quantum-well light-emitting diodes grown on Si substrates by optimizing the InGaN/GaN superlattice interlayer. Opt. Mater. Express 8, 1221 (2018).

15. Sun, Y. et al. Room-temperature continuous-wave electrically pumped InGaN/GaN quantum well blue laser diode directly grown on Si. Light Sci. Appl. 7, (2018).

16. Zuniga-Perez, J. et al. Patterned silicon substrates: a common platform for room temperature GaN and ZnO polariton lasers. Appl. Phys. Lett. 104, 241113 (2014).

17. Damilano, B. et al. Growth of nitride-based light emitting diodes with a high-reflectivity distributed Bragg reflector on mesapatterned silicon substrate: growth of nitride-based LEDs with a high-reflectivity DBR. Phys. Status Solidi A 212, 2297-2301 (2015).

18. Comyn, R. et al. AlGaN/GaN/AlGaN DH-HEMTs grown on a patterned silicon substrate. Phys. Status Solidi A 1700642 (2017). https://doi.org/10.1002/pssa.201700642.

19. Xu, J. et al. Cathodoluminescence study of InGaN/GaN quantum-well LED structures grown on a Si substrate. J. Electron. Mater. 36, 1144-1148 (2007).

20. Mukai, T. Recent progress in group-III nitride light-emitting diodes. IEEE J. Sel. Top. Quantum Electron. 8, 264-270 (2002).

21. Krames, M. R. et al. Status and future of high-power light-emitting diodes for solid-state lighting. J. Disp. Technol. 3, 160-175 (2007).

22. Gil, B. Physics of Wurtzite nitrides and oxides: passport to devices. (Springer, 2014).

23. Hums, C. et al. Fabry-Perot effects in InGaN/GaN heterostructures on Si-substrate. J. Appl. Phys. 101, 033113 (2007).

24. Vurgaftman, I. \& Meyer, J. R. Band parameters for nitrogen-containing semiconductors. J. Appl. Phys. 94, 3675 (2003).

25. Hossain, T. GaN based structures on patterned silicon substrate: Stress and strain analysis. (Nice-Sophia Antipolis, 2012).

26. Even, A. et al. Enhanced In incorporation in full InGaN heterostructure grown on relaxed InGaN pseudo-substrate. Appl. Phys. Lett. 110, 262103 (2017).

27. Pasayat, S. S. et al. Fabrication of relaxed InGaN pseudo-substrates composed of micron-sized pattern arrays with high fill factors using porous GaN. Semicond. Sci. Technol. 34, 115020 (2019).

28. Frayssinet, E., Cordier, Y., Schenk, H. P. D. \& Bavard, A. Growth of thick GaN layers on 4-in. and 6-in. silicon (111) by metalorganic vapor phase epitaxy. Phys. Status Solidi C 8, 1479-1482 (2011).

29. Cliff, G. \& Lorimer, G. W. The quantitative analysis of thin specimens. J. Microsc. 103, 203-207 (1975). 


\section{Acknowledgements}

This work was partly funded by the French National Research Agencey (ANR) within the project no. 2011 RMNP 01801 "MOSAIC." The work done on the NanoCharacterisation PlatForm (PFNC), was supported by the "Recherches Technologiques de Base" Program of the French Ministry of Research. The authors acknowledge the support from the OPTITEC, MINALOGIC, and MONT BLANC INDUSTRIES competitiveness clusters. The authors would like to thank Julien Brault, Mathieu Leroux and Jean Massies for the critical reading of the manuscript.

\section{Author contributions}

B.D. conceived the experimental work with the contribution of G.F. and D.T., participated to the MOVPE growth of the samples and carried out the photoluminescence experiments. E.F. carried out the MOVPE growth of the samples. M.P. carried out the cathodoluminescence experiments. V.B. carried out the scanning electron microscopy experiments. F.F. and C.L. prepared the patterned silicon substrates. D.C. carried out the transmission electron microscopy experiments. B.D. wrote the main manuscript text with the contribution of D.C., M.P., and C.L. All the authors discussed the results and commented on the manuscript during the manuscript writing.

\section{Competing interests}

The authors declare no competing interests.

\section{Additional information}

Supplementary information is available for this paper at https://doi.org/10.1038/s41598-020-76031-3.

Correspondence and requests for materials should be addressed to B.D.

Reprints and permissions information is available at www.nature.com/reprints.

Publisher's note Springer Nature remains neutral with regard to jurisdictional claims in published maps and institutional affiliations.

(c) (i) Open Access This article is licensed under a Creative Commons Attribution 4.0 International License, which permits use, sharing, adaptation, distribution and reproduction in any medium or format, as long as you give appropriate credit to the original author(s) and the source, provide a link to the Creative Commons licence, and indicate if changes were made. The images or other third party material in this article are included in the article's Creative Commons licence, unless indicated otherwise in a credit line to the material. If material is not included in the article's Creative Commons licence and your intended use is not permitted by statutory regulation or exceeds the permitted use, you will need to obtain permission directly from the copyright holder. To view a copy of this licence, visit http://creativecommons.org/licenses/by/4.0/.

(C) The Author(s) 2020 